\title{
Difficult serial anticipation learning in rats: Rule-encoding vs. memory
}

\author{
E. J. CAPALDI, TIMOTHY M. NAWROCKI, and DONNA R. VERRY \\ Purdue University, West Lafayette, Indiana
}

\begin{abstract}
Rats, trained in a runway, were asked to anticipate, while running slowly, the last two events in repeating series of $.045 \mathrm{~g}$ food pellets. The series were either weakly monotonic $(14,5,5,1$, and then 0 pellets/run) or nonmonotonic (5-5-14-1-0). While the terminal 0-pellet event was better anticipated in the weakly monotonic series, the reverse was the case for the next-to-last 1-pellet event. These findings were expected from a memory-discrimination learning hypothesis of serial learning, which suggests that the memory of one event in a series can be used to signal the next event. However, the better anticipation of the 1-pellet event by the nonmonotonic group was inconsistent with the recently stated rule-encoding position of Hulse (1980). According to that view, difficult series of the sort employed in the present investigation are learned by encoding the rule structure of the series, with events in the series with the simple rule structure (the weakly monotonic series in this investigation) being better anticipated than events in the series with the complex rule structure.
\end{abstract}

In this investigation, rats learned to anticipate, as measured by running speed in a runway, different quantities of $.045-\mathrm{g}$ food pellets organized into fiveelement serial patterns. An example of such a pattern is the monotonic decreasing series of food pellets, 14-7-3-1-0, employed by Hulse and Dorsky (1977). On the first run of the 14-7-3-1-0 series, the rat received 14 pellets in the goalbox, on the next run, it received 7 pellets, and so on until, on the fifth, or last, run, 0 pellets were given. The runs of the series were separated by a 10-15-sec interval, and, following the last run, a 10-15-min interval elapsed before the entire series was given again. In the 14-7-3-1-0 series, the 0 -pellet event was anticipated, eventually producing slow running. The present investigation represents a further attempt to test two hypotheses that have been proposed to explain such anticipatory responding, the rule-encoding hypothesis (e.g., Hulse, $1978)$ and the memory-discrimination learning hypothesis (e.g., Capaldi \& Molina, 1979).

According to Hulse (1978; see also Hulse \& Dorsky, 1977, 1979), rule-encoding models, which have been successfully applied to human serial learning, can be equally fruitful when applied to animals which, like people, learn serial tasks by encoding the rule structure. Hulse's aim, essentially, has been to demonstrate for animals what has previously been demonstrated for people. Rule-encoding models suggest, with human data support, that series are learned by the encoding of their rule structure: the simpler the

This research was supported by NSF Grant BNS 80-01171 to E. J. Capaldi. Requests for reprints should be sent to E. J. Capaldi, Department of Psychological Sciences, Purdue University, West Lafayette, Indiana 47907. rule structure, the faster the encoding. Series are said to be more complex as they depart from monotonicity. Confirmation of these propositions was supplied by Hulse and Dorsky (1977), who reported faster anticipation in rats of 0 pellets in the strongly monotonic series, 14-7-3-1-0, than in either the strongly nonmonotonic series, 14-1-3-7-0, or the weakly monotonic series, 14-5-5-1-0 (see also Hulse \& Dorsky, 1979).

According to Capaldi and Molina (1979; see also Capaldi, 1979; Capaldi, Verry, \& Davidson, 1980a), the principles that have been found in discrimination learning generally should apply to a serial learning task, which is seen as a variety of discrimination learning. Specifically, it is suggested that (1) each event in a series can produce a memory which signals the next event, (2) memories signaling rewards equal to or larger than the average of the series are $\mathrm{S}+$ cues and thus produce fast running, (3) memories signaling rewards smaller than the average of the series are S- cues and so produce slow running (see, e.g., Capaldi, 1974, and Haggbloom, 1981), and (4) S+ memories supply the $\mathrm{S}$ - memories with a generalized capacity to signal reward thus elevating speed and reducing anticipatory responding. In the series 14-73-1-0, the memory of 14 pellets, $S^{14}$, signals 7 pellets, $S^{7}$ signals 3 pellets, $S^{3}$ signals 1 pellet, and $S^{1}$ signals 0 pellets. An implication of this view is that anticipatory responding should decrease as the discrimination problem becomes more difficult. Results of this sort, which, it seems, cannot be explained by a ruleencoding view, were reported by Capaldi et al. (1980a). For example, anticipatory responding was found to decrease as the similarity of the $\mathrm{S}+$ and $\mathrm{S}$ - cues increased, a result that was unrelated either to the de- 
gree of monotonicity of the series or the number of instances of the rule contained in the series.

Hulse (1980) made no attempt to explain these findings. However, he provided a more detailed and elaborate statement of his view, which suggested that the findings were irrelevant to the rule-encoding position rather than inconsistent with it, because easy, rather than difficult, serial learning tasks were employed. Hulse (1980) suggested that humans encode the rule structure of a series only if it is difficult to learn, with easy series being learned associatively. According to Hulse's (1980) recent statement, then, two distinct theories are required to deal with serial learning: a cognitive rule-encoding model for difficult series and an associative model for easy series. Recently, Fountain and Hulse (Note 1) have provided data purporting to show that this dual theoretical approach is as necessary for rats as it is for people. Examples of variables, which, according to Hulse (1980), rendered the serial tasks employed by Capaldi and Molina (1979) and Capaldi et al. (1980a) easier than those employed in his laboratory include: series consisting of too few events $(2,3$, and 4 rather than 5); too slow a presentation of the series events (4 min rather than 10-15 sec between runs); too long an interval between series presentations (30 min rather than 10-15 $\mathrm{min})$; and too few series presentations each day ( 1 or 2 rather than 4 ).

The present investigation employed series which would be defined by Hulse as difficult. We sought to determine whether such learning of difficult series would be better described by the rule-encoding model or the memory-discrimination learning model. Whenever possible, levels of variables previously employed by Hulse and Dorsky (1977) were used; indeed, one of the series they employed was used here. In one major respect, however, the present investigation differed from previous ones: it was concerned with anticipation not merely of the last event in the series, but of the last two events. The weakly monotonic series 14-5-5-1-0, previously employed by Hulse and Dorsky (1977), was compared with the nonmonotonic series 5-5-14-1-0. It is interesting to note that when Hulse and Dorsky (1977) employed the series 14-5-5-1-0, the last event in the series was anticipated relatively early in training, with the next to the last event not being anticipated by the time training was terminated. In any event, if Hulse's (1980) recent statement is correct, then the last two events should be better anticipated in the less complex, weakly monotonic series 14-5-5-1-0 than in the more complex nonmonotonic series 5-5-14-1-0. However, if the memory-discrimination learning view is as applicable to such "difficult" serial tasks as it has proved to be to "easy" serial tasks, it may be expected that, while the last event will be better anticipated in the weakly monotonic series, the next to last event will be better anticipated in the nonmonotonic series, thus disconfirming the rule-encoding view.

\section{METHOD}

\section{Subjects}

The subjects were eight naive male albino rats, about 80 days old upon arrival at the laboratory, purchased from the Holtzman Co., Madison, Wisconsin.

\section{Apparatus}

A straight gray runway, $208.4 \mathrm{~cm}$ long, $11.4 \mathrm{~cm}$ high, and $22.9 \mathrm{~cm}$ high, was used. It was covered by wire mesh on a hinged frame. The initial $19.4 \mathrm{~cm}$ of the runway, closed off by an aluminum guillotine door, formed the startbox. Raising the startbox door activated the first of three .01 -sec clocks. Interruption of a photobeam $5.7 \mathrm{~cm}$ from the startbox door stopped the first clock (start time) and started the second clock. Interruption of a second photobeam $133 \mathrm{~cm}$ from the first stopped the second clock (run time) and started the third clock. Interruption of a third photobeam $33 \mathrm{~cm}$ from the second and $6.7 \mathrm{~cm}$ in front of a removable brass insert stopped the third clock (goal time). The brass insert contained a $4.4 \times 3.2 \times 1.6 \mathrm{~cm}$ food cup in which pellets were placed on rewarded trials. An aluminum guillotine door, lowered when the rat broke the third photobeam, confined the rat to the last $30 \mathrm{~cm}$ of the runway, which served as the goalbox.

\section{Procedure}

Upon arrival at the laboratory, the rats were individually caged and given food and water ad lib for 50 days. Day 1 of pretraining began deprivation, which consisted of $13 \mathrm{~g}$ of Wayne Lab Blox each day minus any food given either in pretraining or in the apparatus in experimental training. Water was always available in the home cage. The rats were handled for 1 min on Days 1-8 of pretraining. They were given $1 \mathrm{~min}$ of unbaited runway exposure on Days 9 and 10. They were also given $14.045-\mathrm{g}$ Noyes pellets in their home cages on Days 8-10.

Experimental training lasted 13 days. On Day 1, the rats were divided into two groups of four each. Group WM received the weakly monotonic series of five events 14-5-5-1-0, while Group N received the nonmonotonic series of five events 5-5-14-1-0. The rats were divided into two squads of four each, two from Group WM and two from Group N. They were brought into the experimental room in squads. Squad 1 was always run before Squad 2, but the order of the running of the rats within a squad was randomized daily. Each series was conceptualized as a trial consisting of five runs. Between runs, the rats were confined to a holding cage in which water was always available. Each rat received all five runs of the series before the next rat was run. The animals were run in rotation until each had received four repetitions of its series. The interseries interval was never allowed to drop below $10 \mathrm{~min}$ or to exceed $15 \mathrm{~min}$. The interval elapsing between the runs of the series varied between 10 and $15 \mathrm{sec}$, being determined by how rapidly the experimenter completed the necessary activities, such as reading the clocks and baiting the goalcup.

A run began with removal of the rat from the holding cage and its placement in the startbox. Approximately $3 \mathrm{sec}$ later, the startbox door was raised. The rat was given a maximum of $60 \mathrm{sec}$ to traverse each alley section, for a total time of $180 \mathrm{sec}$. If it did not succeed in traversing an alley section in the allotted $60 \mathrm{sec}$, a time of $60 \mathrm{sec}$ was assigned to that section and the remaining time was added to time used in the next alley section. If a total time of $180 \mathrm{sec}$ elapsed without the rat's reaching the goal, the animal was removed and placed gently in the goalbox. The rat was removed from the goalbox after it had eaten all of the food pellets on rewarded runs or after $15 \mathrm{sec}$ on nonrewarded runs. Each squad was fed the daily ration in the home cage about $15 \mathrm{~min}$ after it had completed runway training.

\section{RESULTS}

All times in all alley sections were converted to speeds $(\mathrm{cm} / \mathrm{sec})$. Only total speeds are reported 
here, however, as anticipatory responding differed little over the different alley sections. Speeds increased in both groups over the initial 5 days of training, and on Day 6 Group WM, but not Group N, showed evidence of anticipating the terminal 0-pellet event. Figure 1 shows running speeds in 2-day blocks for Days 6-13 of training. The speeds shown in each block are for each of the five runs of each series in the order in which the runs were given.

On each of the first two blocks of trials shown in Figure 1, Group WM showed better anticipation of the terminal 0-pellet event than Group N, but over the subsequent two blocks this difference became progressively smaller, and ultimately, negligible. As for the next-to-last event in the series, over the first three blocks of trials shown in Figure 1, the 0-pellet event was anticipated about equally well (or poorly) by both groups, but on the final block of training trials, it was anticipated better by Group $\mathrm{N}$ than by Group WM.

Analysis of variance revealed that only on Block 2 was the difference between the groups significant $[F(1,6)=6.53, p<.05]$. Differences due to runs were significant on all four blocks shown in Figure 1, the smallest difference $[F(4,24)=10.62, p<.001]$ being obtained on the first block of trials. The groups $x$ runs interaction was significant on the first two blocks of trials shown in Figure $1[F(4,24)=3.02$ and 4.03, respectively, $\mathrm{ps}<.05$ ], due mainly to slower running by Group WM on the terminal trial. The groups $\times$ runs interaction was significant on the last block of trials shown in Figure $1[\mathrm{~F}(4,24)=10.10, \mathrm{p}<.001]$, due mainly to slower running by Group $\mathrm{N}$ on the next to the last run. On the third block of trials shown in Figure 1, the groups $\times$ runs interaction was not significant $[F(4,24)=2.07, p>.05]$, indicating that this block was a transition in which Group N performed about as well as Group WM on the terminal run, but not as yet showing better anticipation on the next-to-last run. Additional points of information became available by breaking down the groups $x$ runs interaction with Newman-Keuls posttests.

Posttests revealed that on each of the four blocks

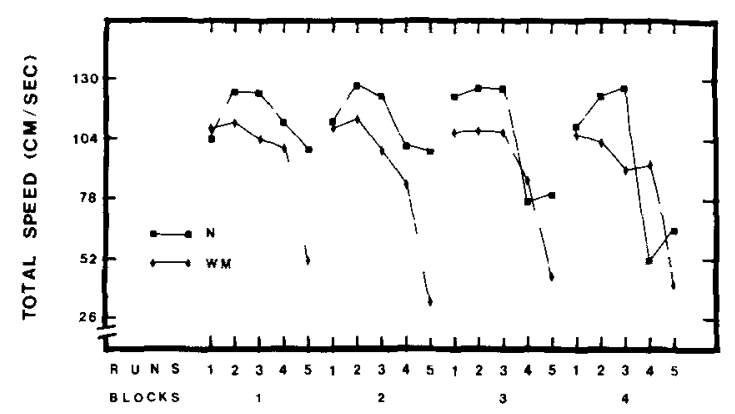

Figure 1. Mean total speed $(\mathrm{cm} / \mathrm{sec})$ in blocks of 2 days for Groups $N$ and WM on Days 6-13 of training on each of the five runs of each of the patterns, 5-5-14-1-0 and 14-5-5-1-0. shown in Figure 1, Group WM ran more slowly on its terminal 0-pellet run than on all other runs (all ps $<.01$ ), with no other difference being significant. On a statistical basis, then, Group WM failed to anticipate the next-to-last trial in the series at any point in training. Group $\mathrm{N}$ anticipated the last two runs in the series on the last block of training trials (ps $<.05$ or better). Differences between the groups, as revealed by the posttests, were as follows: Group WM ran more slowly than Group $\mathbf{N}$ on the terminal run on each of the first two blocks of trials shown in Figure 1 (ps $<.05$ ), and Group $N$ ran more slowly than Group WM on the next-to-last run of Block 4 $(p<.05)$, with no other differences between the groups being significant.

\section{DISCUSSION}

The weakly monotonic series of Group WM, 14-55-1-0, produced much the same result here as in the Hulse and Dorsky (1977) investigation: the last event in the series was anticipated relatively early in training, but the next-to-last event was not anticipated by the time training was terminated. Hulse and Dorsky (1977) used 11 days of training. The slightly more extensive training employed here, 13 days, clearly supports their finding that anticipation of the nextto-last event was a most difficult task for Group WM.

Group N, which received the nonmonotonic series, 5-5-14-1-0, did anticipate the next-to-last event, while requiring more training than Group WM to anticipate the last event. These findings are clearly incompatible with a major and fundamental implication of Hulse's rule-encoding view, the proposition that all events in a series with a relatively simple rule structure should be relatively easy to anticipate, while all events in a series with a more complex rule structure should be relatively difficult to anticipate. On the contrary, the present results indicate that events in a series with the simpler rule structure may be more, as well as less, difficult to anticipate than events in a series with the more complex rule structure. Therefore, an emphasis upon rule structure alone, without reference to some additional factor or factors, cannot possibly explain the present findings.

The view that serial learning findings reported by Capaldi and Molina (1979) and Capaldi et al. (1980a) are irrelevant to the rule-encoding view because easy series were employed seems highly problematical on the basis of the results obtained here. Essentially, the "difficult"' series employed here produced results highly compatible with the earlier findings said to result from "easy" series. Thus, two distinct theories would not seem to be required to deal with available serial learning data. Indeed, a memory-discrimination learning view, previously shown by Capaldi et al. (1980a; see also Capaldi, Verry, \& Davidson, 1980b) to be compatible with all available serial learning 
findings, including those from Hulse's laboratory, can be shown to be compatible with the present findings as well.

The present view is that anticipation of the nextto-last event involved a more difficult discrimination problem for Group WM than Group N, with the reverse being the case for the last event. In Group WM, which received the series 14-5-5-1-0, the next-to-last event was signaled by memory of the previous 5-pellet event, $S^{5}$. However, $S^{5}$ was an $S+$ cue as well, signaling the third event of the series, a 5-pellet event. Thus, Group WM had the difficult task of deciding when $S^{5}$ signaled 5 pellets (run fast) and when $S^{5}$ signaled 1 pellet (run slowly), and thus anticipatory responding on the next-to-last event failed to develop by the time training was terminated. In contrast, Group $\mathrm{N}$ had an easy discrimination in connection with the next-to-last event. For Group N, which received the series 5-5-14-1-0, the next-to-last event was signaled by $\mathrm{S}^{14}$, a memory quite discriminable from the $S+$ cue of the series, $S^{5}$.

In both series, the last event was signaled by $\mathrm{S}^{\mathbf{1}}$, and in both series the most similar $\mathrm{S}+$ cue supplying $S^{1}$ with a generalized capacity to signal reward was $\mathrm{S}^{5}$. A major factor here, it is suggested, was that $\mathrm{S}^{5}$ signaled a much larger reward in the 5-5-14-1-0 series, 14 pellets, than in the 14-5-5-1-0 series, only 5 pellets. This being so, $S^{5}$ supplied $S^{1}$ with a greater generalized capacity to signal reward in Group $N$ than in Group WM, providing the latter group with the more difficult discrimination problem (see Capaldi et al., 1980a).

According to Hulse (1978; see also Hulse \& Dorsky, 1977, 1979), in human serial learning investigations, an emphasis upon rule structure alone, without recourse to other factors, has proved sufficient to explain both serial anticipation learning and transfer in serial tasks. Clearly, there is a major discrepancy between human serial learning data, as characterized by Hulse and associates, and the animal serial learning data reported here and by Capaldi and Molina (1979) and Capaldi et al. (1980a). This might suggest to some that rats do not encode rules, that ruleencoding models are applicable to people but not to rats, and that Hulse and his associates are simply wrong. On the other hand, further human research may reveal that serial learning in people, as in rats, cannot be predicted from a knowledge of rule structure alone. After all, there is a strong human serial learning tradition, human verbal learning being a case in point, which emphasizes associations between the events of a series, or between an event and its position in the series, and so on (see, e.g., Crowder, 1976). Hulse and his associates, we would suggest, have focused upon one tradition in human serial learning, that which emphasizes rule-ordered serial learning. Perhaps, if Hulse's (1980) latest position that rats employ either associative mechanisms (easy serial tasks) or cognitive rule-encoding mechanisms (difficult serial tasks) were broadened to include the notion that all serial learning is some blending of both, a more viable approach to rule-encoding in animals might result.

\section{REFERENCE NOTE}

1. Fountain, S. B., \& Hulse, S. H. Pattern length and complexity determine serial learning in rats. Paper presented at the Eastern Psychological Association, New York, May 1981.

\section{REFERENCES}

Capaldi, E. J. Partial reward either following or preceding consistent reward: A case of reinforcement level. Journal of Experimental Psychology, 1974, 102, 954-962.

CAPAldi, E. J. Latent discrimination learning under a regular schedule of partial reinforcement. Animal Learning \& Behavior, $1979,7,63-68$

Capaldi, E. J., \& Molina, P. Element discriminability as a determinant of serial-pattern learning. Animal Learning \& Behavior, 1979, 7, 318-322.

Capaldi, E. J., Verry, D. R., \& Davidson, T. L. Memory, serial anticipation pattern learning, and transfer in rats. Animal Learning \& Behavior, 1980, 8, 575-585. (a)

Capaldi, E. J., Verry, D. R., \& Davidson, T. L. Why ruleencoding by animals in serial learning remains to be established. Animal Learning \& Behavior, 1980, 8, 691-692. (b)

Crowder, R. G. Principles of learning and memory. Hillsdale, N.J: Erlbaum, 1976.

HAGGBLOOM, S. J. Effects of reinforcement level and number of $N-R$ transitions on resistance to discrimination. Bulletin of the Psychonomic Society, 1981, 17, 197-199.

Hulse, S. H. Cognitive structures and serial-pattern learning by rats. In S. H. Hulse, H. Fowler, \& W. K. Honig (Eds.), Cognitive processes in animal behavior. Hillsdale, N.J: Erlbaum, 1978.

Hulse, S. H. The case of the missing rule: Memory for reward vs. formal structure in serial-pattern learning by rats. Animal Learning \& Behavior, 1980, 8, 689-690.

Hulse, S. H., \& Donsky, N. P. Structural complexity as a determinant of serial-pattern learning. Learning and Motivation, $1977,8,488-506$.

Hulse, S. H., \& Dorsky, N. P. Serial-pattern learning by rats: Transfer of a formally defined stimulus relationship and the significance of nonreinforcement. Animal Learning \& Behavior, 1979, 7, 211-220.

(Manuscript received June 29, 1981; revision accepted for publication November 21,1981 .) 